\title{
Hybrid Inorganic-Metalorganic Compounds Containing Copper(II)- Monosubstituted Keggin Polyanions and Polymeric Copper(I) Complexes.
}

Leire San Felices, Pablo Vitoria, Juan M. Gutiérrez-Zorrilla, Luis Lezama and Santiago Reinoso

\section{Supporting Information}

Table of Contents

Fig. S1. Q-band single crystal EPR spectrum of compound 1 measured at three different rotation angles in an arbitrary axis.

Fig. S2. DFT calculated IR spectra of complete and copper(II) monosubstituted Keggin anions.

Table S1. Listings of continuous symmetry measures (CSM) for all entries retrieved from the CSD database. 
Fig. S1. Q-band single crystal EPR spectrum of compound 1 measured at three different rotation angles in an arbitrary axis.

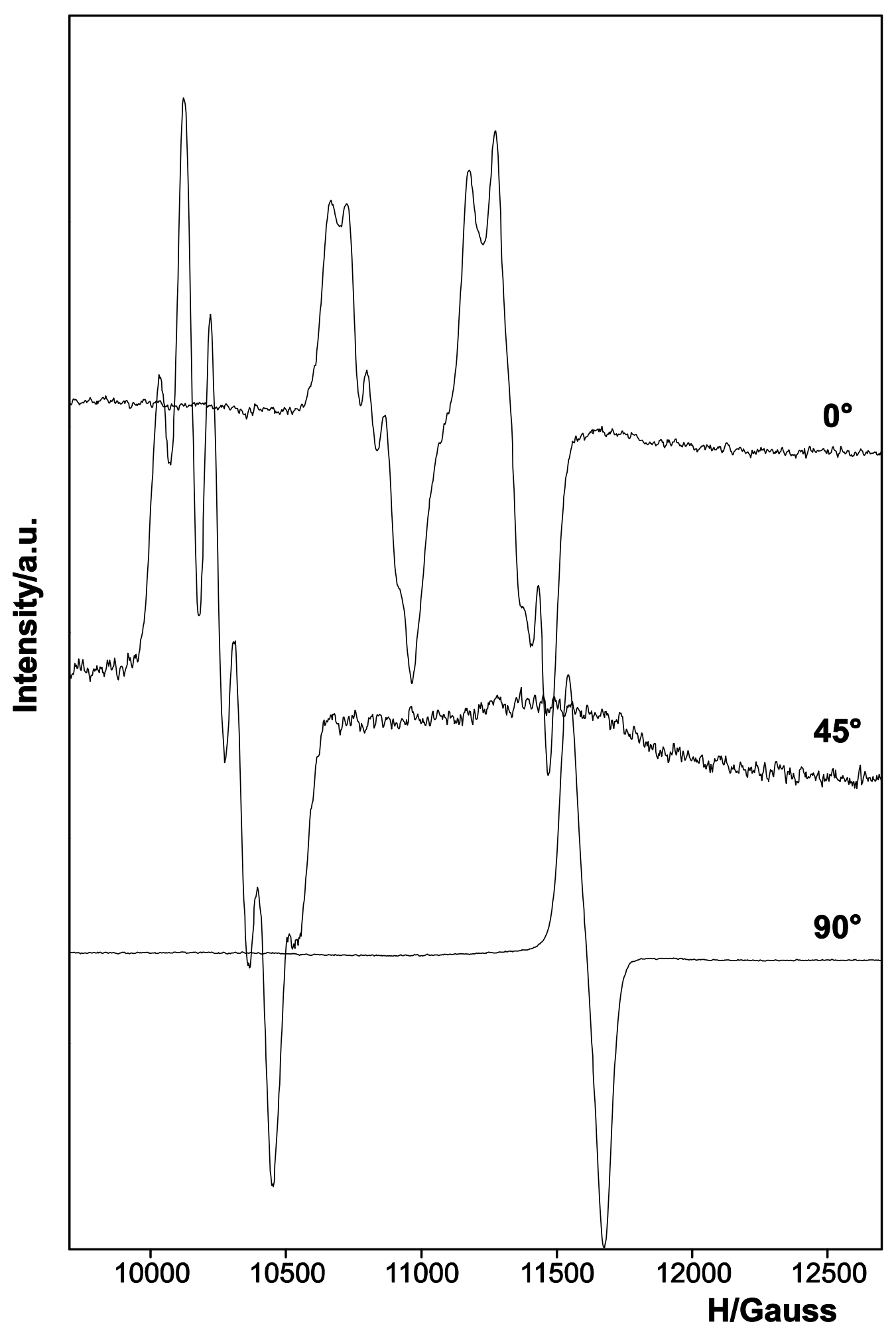


Fig. S2. DFT calculated IR spectra of complete and copper(II) monosubstituted Keggin anions.

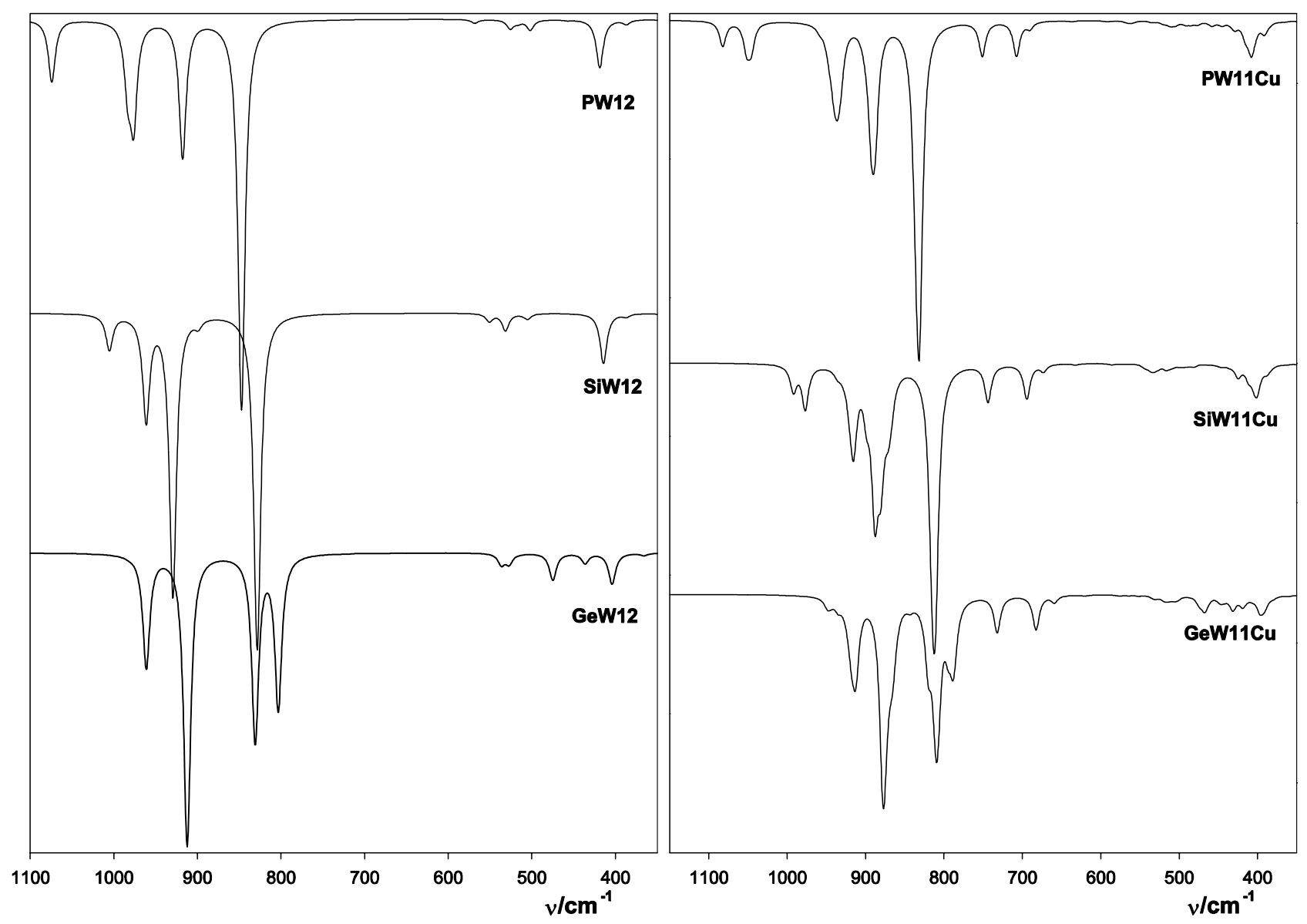


Table S1. Listings of continuous symmetry measures (CSM) for all entries retrieved from the CSD database.

\begin{tabular}{|c|c|c|c|}
\hline Structure & [ML3] & $\mathrm{S}(\mathrm{VT}-3)$ & $S(T P-3)$ \\
\hline BAFCUH & & 6.31556 & 3.85486 \\
\hline BAHMON & & 6.78623 & 4.83049 \\
\hline BEVKOD & & 5.33783 & 3.14229 \\
\hline EYIMEE & & 5.92195 & 3.10172 \\
\hline FAXQOK & & 3.54587 & 1.84929 \\
\hline FIFNAK_1 & & 6.34738 & 3.99303 \\
\hline FIFNAK_2 & & 6.18158 & 3.50885 \\
\hline FOCLOZ & & 3.13283 & 0.88156 \\
\hline HECYUJ & & 5.22029 & 2.32671 \\
\hline JUXVUT & & 5.42179 & 3.21603 \\
\hline JUXWAA & & 6.31186 & 3.90900 \\
\hline MASQON_1 & & 6.85186 & 5.69964 \\
\hline MASQON_2 & & 7.69480 & 5.47323 \\
\hline MASQON_3 & & 9.11488 & 7.12403 \\
\hline $\mathrm{MASQON}^{-} 4$ & & 10.26374 & 8.33247 \\
\hline SAZPEO & & 11.65129 & 9.59191 \\
\hline UFUTOE & & 9.64120 & 7.64350 \\
\hline VELHUP & & 7.12784 & 4.73773 \\
\hline WUVBUK & & 5.00232 & 2.98213 \\
\hline XONHAJ & & 5.67042 & 2.95752 \\
\hline XOSZAG & & 5.81302 & 3.44340 \\
\hline YASHEG & & 5.08386 & 2.81498 \\
\hline ZEBCOY & & 3.04759 & 1.93457 \\
\hline $11-3$ & $\mathrm{C} 3 \mathrm{v}$ & \multirow{2}{*}{\multicolumn{2}{|c|}{ Vacant tetrahedron }} \\
\hline $\mathrm{TP}-3$ & D3h & & \\
\hline
\end{tabular}

\begin{tabular}{|c|c|c|c|c|}
\hline Structure & [ML4 ] & $S(S W-4)$ & $S(T-4)$ & $S(S P-4)$ \\
\hline CEJQOX02 & & 3.58309 & 2.93064 & 26.91822 \\
\hline CUACPY & & 12.90114 & 24.07367 & 1.27468 \\
\hline CUACPYO1 & & 12.89963 & 24.08702 & 1.18455 \\
\hline DAYZAE & & 9.30131 & 10.37451 & 31.11939 \\
\hline ESEHOZ & & 19.10354 & 33.52334 & 0.28501 \\
\hline ESUBID & & 3.81598 & 3.17208 & 29.61084 \\
\hline FANZEA & & 3.46228 & 4.27918 & 32.80921 \\
\hline FEXVUA & & 5.48966 & 1.18183 & 30.39694 \\
\hline FOCLIT & & 3.01514 & 3.38819 & 29.77126 \\
\hline FOCLUF & & 4.64796 & 3.39710 & 27.91834 \\
\hline GONRUW & & 2.01142 & 4.79013 & 23.36001 \\
\hline GONSAD & & 6.23677 & 1.49015 & 30.22429 \\
\hline $\mathrm{HAKCOM}$ & & 18.90791 & 38.66252 & 7.99378 \\
\hline HAYFES 1 & & 15.26915 & 28.54662 & 0.35541 \\
\hline HAYFES ${ }^{-} 2$ & & 14.42545 & 27.53227 & 0.47173 \\
\hline HAYFIW 1 & & 13.31358 & 25.93767 & 0.77041 \\
\hline HAYFIW-2 & & 14.63904 & 27.63037 & 0.49511 \\
\hline JODGAK $_{-}^{-1}$ & & 12.58912 & 23.25010 & 1.60756 \\
\hline JODGAK $^{-} 2$ & & 8.82414 & 18.94183 & 2.81935 \\
\hline JOWJEK ${ }^{-}$ & & 19.59536 & 37.18474 & 10.20334 \\
\hline JUXWEE & & 11.27533 & 9.00787 & 30.07594 \\
\hline MEPCUA & & 18.75976 & 33.35048 & 0.02573 \\
\hline MEPCUC & & 18.83696 & 33.33774 & 0.00661 \\
\hline MIDVEA & & 19.07325 & 33.53669 & 0.30602 \\
\hline MIDVOK & & 18.99085 & 33.52770 & 0.29267 \\
\hline OGEJAL & & 3.22784 & 5.25469 & 19.39528 \\
\hline OGEJEP 1 & & 8.09989 & 12.47985 & 10.27694 \\
\hline OGEJEP_2 & & 10.58949 & 10.13107 & 31.28564 \\
\hline PIDZIL_-1 & & 10.30793 & 19.88484 & 2.69308 \\
\hline PIDZIL_-2 & & 14.00038 & 26.22676 & 0.65820 \\
\hline PIJCEQ & & 2.15458 & 4.96997 & 28.75969 \\
\hline RIDSAY_1 & & 10.42692 & 19.56014 & 3.00898 \\
\hline RIDSAY ${ }^{-} 2$ & & 11.64398 & 22.51038 & 1.52814 \\
\hline $\mathrm{RUXHOH}$ & & 8.11526 & 7.26095 & 24.79129 \\
\hline SAZPEO01 & & 16.82081 & 36.25050 & 4.37658 \\
\hline SOZCUF 1 & & 15.16638 & 28.00270 & 0.44102 \\
\hline SOZCUF $^{-} 2$ & & 15.20288 & 27.85484 & 0.46814 \\
\hline UFUTIY $^{-} 1$ & & 4.61852 & 3.77334 & 32.48190 \\
\hline UFUTIY_-2 & & 3.95228 & 2.54079 & 30.17878 \\
\hline UFUTOE & & 3.77076 & 2.63571 & 30.88069 \\
\hline VAHCUD & & 18.84564 & 33.34184 & 0.01276 \\
\hline VAQQAG & & 9.79240 & 22.22864 & 4.75142 \\
\hline VAQQIO_1 & & 5.27134 & 6.55791 & 17.63009 \\
\hline VAQQIO_-2 & & 10.33570 & 24.35715 & 5.23727 \\
\hline WAJDOB & & 6.15404 & 3.37831 & 31.49903 \\
\hline WAJDOB01 & & 6.14996 & 3.24630 & 31.86039 \\
\hline WUVBUK & & 19.19587 & 33.49173 & 0.23760 \\
\hline XOZXIT_1 & & 7.37899 & 9.78311 & 16.94274 \\
\hline XOZXIT $^{-} 2$ & & 7.93048 & 9.60945 & 19.26913 \\
\hline $\mathrm{xozxOz}^{-1}$ & & 7.42216 & 9.68346 & 17.62551 \\
\hline $\mathrm{xozxOZ}^{-} 2$ & & 7.68548 & 9.58311 & 18.85142 \\
\hline XUPCUG & & 6.13423 & 5.50486 & 29.75820 \\
\hline ZIRHUD 1 & & 10.63807 & 20.29229 & 2.67840 \\
\hline ZIRHUD 2 & & 13.72829 & 25.75623 & 0.72868 \\
\hline ZIRJAL $^{-} 1$ & & 10.80860 & 20.29651 & 2.59926 \\
\hline ZIRJAL 2 & & 14.00343 & 25.86369 & 0.70523 \\
\hline ZIRJEP 1 & & 10.88253 & 20.41620 & 2.63631 \\
\hline ZIRJEP_2 & & 13.47985 & 25.35810 & 0.79043 \\
\hline & & \multicolumn{2}{|c|}{ Sawhorse } & \\
\hline & $\mathrm{Td}$ & \multicolumn{2}{|c|}{ Tetrahedron } & \\
\hline$P-4$ & $\mathrm{D} 4 \mathrm{~h}$ & Square & & \\
\hline
\end{tabular}

